\title{
Population Balance Modeling of Growth and Secondary Nucleation by Attrition and Ripening
}

\section{Journal Article}

Author(s):

Bosetti, Luca (D); Mazzotti, Marco

Publication date:

2020-01-02

Permanent link:

https://doi.org/10.3929/ethz-b-000391759

Rights / license:

In Copyright - Non-Commercial Use Permitted

Originally published in:

Crystal Growth \& Design 20(1), https://doi.org/10.1021/acs.cgd.9b01240 


\title{
Population Balance Modeling of Growth and Secondary Nucleation by Attrition and Ripening
}

\author{
Luca Bosetti ${ }^{(0)}$ and Marco Mazzotti*i) \\ Institute of Process Engineering, ETH Zurich, 8092 Zurich, Switzerland \\ Supporting Information
}

ABSTRACT: Secondary nucleation is ubiquitous in nature and of fundamental importance for both batch and continuous crystallization processes. Attrition is the mechanism through which fragments are formed after the collision of a crystal with a stirrer. Those fine fragments, if small enough, are considered secondary nuclei. In this work, starting from the mechanistic description of attrition by Gahn and Mersmann (Crystallization Technology Handbook; CRC Press, 2001), two population balance equation models to simulate secondary

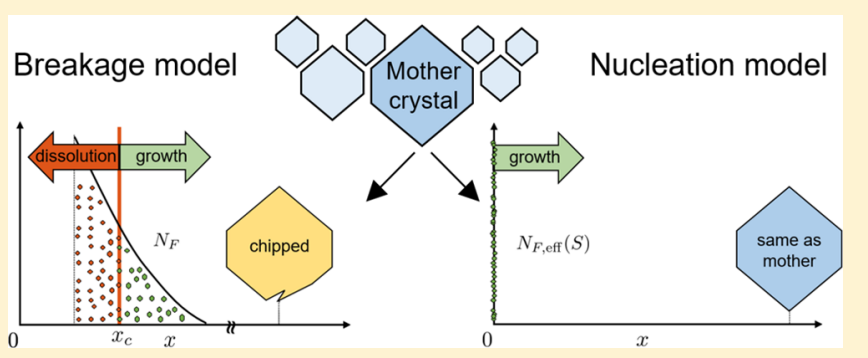
nucleation processes have been derived. The first simulates attrition as a breakage term, and growth rate is the result of size-dependent solubility. The second model considers attrition as a boundary condition at zero crystal size, where the expression for secondary nucleation rate already takes into account the effect of supersaturation, while the growth rate is size-independent. The two models are proven equivalent in the growth regime, thus where secondary nucleation and growth are the dominant phenomena. At extremely low values of supersaturation, thanks to size-dependent solubility, the first model yields to further development of the crystal population, e.g., ripening and aging. The main result is that secondary nucleation by attrition can be described as a birth/death term or, alternatively, as a source term according to the final application of the model. Since the two approaches have very different computational intensities, one can choose the right model based on the objective of the simulation study. The evolution of the crystal population for high values of supersaturation will be the same in both cases.

\section{INTRODUCTION}

Nucleation is a phenomenon ubiquitous both in nature and in manufacturing processes. In particular, secondary nucleation (the formation of new stable crystals, or nuclei, of a solute in the presence of crystals of the same compound) is the mechanism through which virtually all product crystals of continuous crystallization are formed, e.g., in the case of active pharmaceutical compounds. $^{2-4}$ Though it has been shown that secondary nuclei can form by (i) attrition, (ii) shear, or (iii) surface breeding, the level of qualitative and quantitative understanding about the details of these different mechanisms is not satisfactory yet. ${ }^{5}$ As a consequence, current population balance equation (PBE) models account for secondary nucleation, whatever the mechanism, mostly by adding the rate of secondary nucleation to that of primary nucleation, and using the sum (divided by the growth rate) as the boundary condition at zero length for the particle size distribution (PSD). ${ }^{6}$ In this case, the secondary nucleation rate is given by an empirical relationship that depends on the amount of crystals suspended (volume or surface thereof), on the supersaturation level (directly or through the growth rate), and on the extent of mixing (typically through the average energy dissipation); the secondary nucleation rate increases with increasing values of each of these three quantities. ${ }^{7}$

In this contribution, we are going to focus only on the first mechanism, i.e., formation of secondary nuclei by attrition.
Mersmann and co-workers have analyzed in detail secondary nucleation by attrition, and they were able to develop a comprehensive quantitative theory, validated by experiments, ${ }^{8-10}$ of how secondary nuclei form from a single crystal upon collision with the reactor stirrer, and to analyze whether these tiny fragments (fines), once separated by the parent crystal, grow or dissolve. ${ }^{1,11,12}$ Such a first-principles model was then embedded in a PBE framework by Bermingham in his Ph.D. dissertation. ${ }^{13}$ It is worth noting that such description of secondary nucleation by attrition is in principle not consistent with the empirical constitutive equation for its rate that we have summarized above.

In an ongoing effort to improve our understanding and characterization of secondary nucleation, our motivation is that of reconciling the first-principles description of secondary nucleation by attrition as proposed by Mersmann with the population balance model (PBM) framework that is commonly exploited to synthesize, design, and optimize continuous crystallization processes. More specifically, we would like to implement Mersmann's well-established first-principles model of attrition as a source of secondary nuclei, and plug it into a PBE in two different ways, namely, either as a breakage contribution

Received: September 18, 2019

Revised: November 8, 2019

Published: November 12, 2019 
to the $\mathrm{PBE}$, or as a nucleation term, i.e., as a zero-length boundary condition on the PSD. The two approaches have specific challenges, and they exhibit different pros and cons. By addressing the former and elucidating the latter, we will be able to achieve a better insight into secondary nucleation by attrition. With this conceptual and theoretical scope in mind and for the sake of brevity, we have refrained from including in this work any experimental study, especially because Mermann's theory has already been investigated and validated experimentally in the literature. ${ }^{8-10}$

Thus, concluding, the novelty of this manuscript consists of (i) the translation of Mersmann's secondary nucleation by attrition model into two different types of description of secondary nucleation in a PBE, (ii) the development of a combination of efficient numerical methods to solve the PBE in the two cases, (iii) the analysis of similarities and differences in the PBE solution in the two cases, (iv) the application of the two approaches to different exemplary cases, and their final comparative assessment.

\section{PHYSICAL MODEL}

The goal of this work is to describe an isothermal seeded crystallization process from solution, where existing crystals may grow and dissolve (depending on the solution concentration and on the crystal size), and new crystals may form as a consequence of secondary nucleation (by attrition); primary nucleation and agglomeration are assumed to be negligible. We intend to describe secondary nucleation by attrition either explicitly as a breakage phenomenon generating a population of fragments of different sizes (we will call this the "breakage model"), or as a nucleation event yielding new nuclei all of size zero ("nucleation model"). We describe the evolution of the PSD and of the solute concentration through a PBE and a mass balance in solution. In this section, the equations are written in a general form, where both breakage and nucleation are included; then solubility, growth, and dissolution are discussed, and finally the equations are normalized and made dimensionless for the sake of an efficient numerical solution. The following section will deal with the attrition model; hence, the model equations will specialize into either the "breakage model" or the "nucleation model".

Model Equations. The model consists of the following population balance equation and solute mass balance:

$$
\begin{aligned}
& \frac{\partial f}{\partial t}+\frac{\partial(G f)}{\partial x}=B-D \\
& \frac{\mathrm{d} c}{\mathrm{~d} t}=-k_{\mathrm{v}} \rho_{\mathrm{c}} \frac{\mathrm{d} \mu_{3}}{\mathrm{~d} t}
\end{aligned}
$$

In the PBE, $f$ is the PSD, $G$ is the growth rate, $B$ and $D$ are general birth and death terms, and $x$ is the characteristic crystal length. In the mass balance, $c$ is the concentration, $k_{\mathrm{v}}$ the volume shape factor, $\rho_{\mathrm{c}}$ the density of the crystals, and $\mu_{3}$ is the third moment of the PSD defined as

$$
\mu_{3}=\int_{0}^{\infty} x^{3} f(x) \mathrm{d} x
$$

The initial and boundary conditions are

$$
\begin{aligned}
& f(x, t=0)=f_{\text {init }}(x) \\
& c(t=0)=c_{\text {init }} \\
& f(x=0, t)=J / G_{0}
\end{aligned}
$$

where $f_{\text {init }}$ and $c_{\text {init }}$ are the initial PSD and concentration, respectively, $J$ is the nucleation rate, i.e., the number of crystals formed per unit time and unit mass of solution, and $G_{0}$ is the growth rate at size 0 and at time $t$.

The growth rate is in general a function of the solute concentration, $c$, and of the particle size

$$
G=G(c, x)
$$

In the case where the only source terms are due to breakage, their expressions for the crystal of size $x$ at time $t$ can be written as

$$
\begin{aligned}
& B=\int_{x}^{\infty} b(\eta) f(\eta, t) g(x, \eta) \mathrm{d} \eta \\
& D=b(x) f(x, t)
\end{aligned}
$$

where $b$ is the frequency at which breakage events occur, and $g$ is the daughter size distribution, which defines the distribution and the number of fragments generated by breakage.

If we assume primary nucleation is not occurring, the only contribution to the nucleation rate is given by secondary nucleation, whose most general empirical expression can be written as

$$
J_{\mathrm{S}}=E_{\mathrm{t}}(\theta) F_{1}(c) F_{2}(f)
$$

where $E_{\mathrm{t}}$ and $F_{1}$ are generic functions and $\theta$ represents the operating conditions (e.g., the stirring rate); $F_{1}$ accounts for the effect of concentration on the secondary nucleation, e.g., through the growth rate; $F_{2}$ is a functional of the PSD.

In the following sections, all the phenomena already mentioned are going to be fully described, the functional dependencies explained, and the variables are categorized as physical properties, operating conditions, and estimated parameters. The physical properties are usually taken from the literature for potassium alum; the operating conditions are those of a stirred tank reactor, and they are also taken from the literature. The estimated parameters are usually guessed or assumed equal to the same parameters used for similar systems in other works in the literature.

Solubility, Growth, and Dissolution. The formation of a new particle from a supersaturated solution results in a gain in Gibbs free energy proportional to the volume of the newly formed nucleus and a loss related to the creation of its surface. Summing the two Gibbs free energy contributions, one can find the critical nucleus size, $x_{\mathcal{c}}$, above which particles are stable and grow. ${ }^{1,14}$ The critical nucleus size, $x_{c}$, depends on the supersaturation, which is defined as ${ }^{15}$

$$
S=\frac{c}{c_{\infty}}
$$

where $c_{\infty}$ is the bulk solubility, and it can be written as

$$
x_{\mathrm{c}}(S)=\frac{2 k_{\mathrm{a}} \gamma V_{\mathrm{m}}}{3 k_{\mathrm{v}} R T \ln S}=\frac{\alpha}{\ln S}
$$

where $k_{\mathrm{a}}$ and $k_{v}$ are the area and volume shape factors, $\gamma$ the surface tension, $V_{\mathrm{m}}$ the molar volume, $R$ the universal gas constant, $T$ the absolute temperature, and $\alpha$ is called capillary length.

This implies that also the solubility depends on the particle size, and it can be expressed using the Gibbs-Thomson or Gibbs-Kelvin equation as 


$$
S^{*}(x)=\frac{c^{*}(x)}{c_{\infty}}=\exp \left(\frac{\alpha}{x}\right)
$$

The bulk solubility has been experimentally measured for different temperatures and fitted as

$$
c_{\infty}(T)=c_{\mathrm{r}} \exp (\beta T)
$$

with the parameters for capillary length and solubility in Table 2 .

Growth and Dissolution. The driving force for growth and dissolution of a crystal of size $x$ is the difference between the concentration of the solution and its size-dependent solubility value; thus, $G(c, x) \propto c-c^{*}(x)$.

According to Lifshitz and Slyozov ${ }^{16}$ and Wagner, ${ }^{17}$ the growth rate can be formulated using eq 9 as

$$
G(c, x)=k_{\mathrm{g}}\left(\frac{c}{c_{\infty}}-\frac{c^{*}(x)}{c_{\infty}}\right)=k_{\mathrm{g}}\left(S-\exp \left(\frac{\alpha}{x}\right)\right)
$$

where $k_{\mathrm{g}}$ has been defined as

$$
k_{\mathrm{g}}=k_{\mathrm{g}, 1} \exp \left(-\frac{E_{\mathrm{g}}}{R T}\right)
$$

in order to take into account the dependence on temperature, while $k_{\mathrm{g}, 1}$ and $E_{\mathrm{g}}$ are constants to be estimated. Note that in the classical treatment of Ostwald ripening by Lifshitz and Slyozov ${ }^{16}$ and Wagner ${ }^{17}$ (and also in our previous contribution ${ }^{18}$ ), only the first order term in $\alpha / x$ is retained in the exponential term of eq 11 , thus resulting in

$$
G(c, x)=k_{\mathrm{g}}\left(S-\left(1+\frac{\alpha}{x}\right)\right)
$$

For our calculations, the quantitative differences between eq 11 and eq 13 can be neglected.

In the breakage model, the growth rate depends on concentration and size: given a value of $c$, it can be positive for the larger particles, i.e., net growth, and negative for the smaller ones, i.e., net dissolution, according to the value of the critical size, as shown in Figure 1.

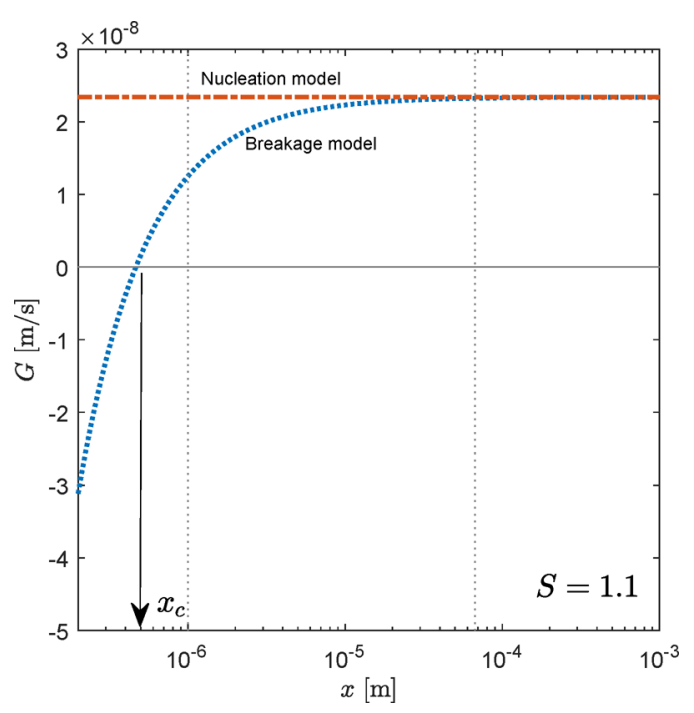

Figure 1. Comparison of the growth rate expressions for the breakage, eq 11, and the nucleation model, eq 14. The critical crystal size corresponds to the change in sign of the growth rate in the model with size-dependent solubility.
In the nucleation model, since the secondary nucleation rate is treated as a boundary condition at size 0 , the size-dependent growth expression in eq 11 cannot be used because it would lead to immediate dissolution of all the new nuclei produced. For this reason, solubility is assumed to be size-independent, i.e., $c^{*}(x)=c_{\infty}$, hence

$$
G(c)=k_{\mathrm{g}}\left(\frac{c}{c_{\infty}}-1\right)=k_{\mathrm{g}}(S-1)
$$

The growth rates of the two models tend asymptotically to the same value, as illustrated in Figure 1. Note that the growth rates have been calculated for the set of physical and estimated parameters reported in Table 2.

Rescaled Model. The model is modified, by introducing the following dimensionless variables and functions, according to the scheme developed by Iggland and Mazzotti ${ }^{18,19}$

$$
\begin{aligned}
& y=\frac{x}{x_{0}} \\
& \tau=\frac{t}{t_{0}} \text { with } t_{0}=\frac{x_{0}}{k_{\mathrm{g}}} \\
& f \mathrm{~d} x=n \mathrm{~d} y \Rightarrow n=f x_{0}
\end{aligned}
$$

where $x_{0}$ is the rescaling factor, i.e., the reference crystal size, which can be chosen arbitrarily, with the goal of having a characteristic dimensionless crystal size close to unity; $t_{0}$ is the reference time, $y$ and $\tau$ are the dimensionless size and time, respectively. The rescaled PSD, $n$, has units of $[\# / \mathrm{kg}]$. With the definitions above, the dimensionless growth rate is newly defined as

$$
\tilde{G}(y)=\frac{G(x)}{k_{\mathrm{g}}}
$$

Substituting these definitions yields the following dimensionless form of the PBE and of the solute mass balance (after division by $c_{\infty}$ ) of eq 1 :

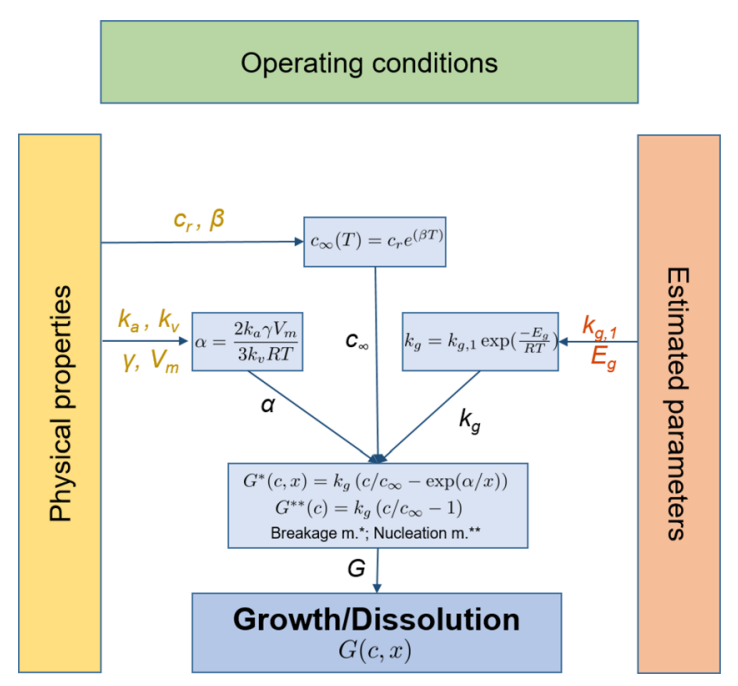

Figure 2. Physical properties, operating conditions, and estimated parameters playing a role in the definition of the growth rate. The kinetic growth is size-dependent only in the case of the breakage model. 


$$
\begin{aligned}
& \frac{\partial n}{\partial \tau}+\frac{\partial(\tilde{G} n)}{\partial y}=\tilde{B}-\tilde{D} \\
& \frac{\mathrm{d} S}{\mathrm{~d} \tau}=-\frac{k_{\mathrm{v}} \rho_{\mathrm{c}}}{c_{\infty}} x_{0}^{3} \frac{\mathrm{d} \phi_{3}}{\mathrm{~d} \tau}
\end{aligned}
$$

where $\phi_{3}$ is the third moment of the rescaled PSD, $n$, calculated as

$$
\phi_{3}=\int_{0}^{\infty} y^{3} n(y) \mathrm{d} y=\frac{\mu_{3}}{x_{0}^{3}}
$$

The rescaled initial and boundary conditions are

$$
\begin{aligned}
& n(y, \tau=0)=n_{\text {init }}(y) \\
& S(\tau=0)=S_{\text {init }} \\
& n(y=0, \tau)=\frac{\tilde{J}}{\tilde{G}_{0}} x_{0}
\end{aligned}
$$

In the equation above, the quantities $\tilde{B}, \tilde{D}$ are the rescaled breakage and death terms, and $J$ is the rescaled nucleation rate, which are defined in the Supporting Information.

\section{MODELING SECONDARY NUCLEATION BY ATTRITION}

Attrition is the mechanism of formation of small fragments resulting from the collision of a particle with a harder body, e.g., the stirrer. Gahn and Mersmann ${ }^{1,20}$ developed a first principle attrition model for brittle crystals. Its validity has been extensively proven by the authors considering a large number of inorganic brittle crystals, e.g., potassium alum, potassium nitrate, potassium sulfate, and many others. ${ }^{11,12}$ Some years later, Bermingham ${ }^{13}$ applied the theory of Gahn and Mersmann by including it in a population balance model.

The model is based on a number of assumptions and general considerations: ${ }^{20}$

- Geometry. The impact force is acting in a normal direction on the corner of the mother crystal, which is considered to be a cone of angle $120^{\circ}$ : the region closest to the corner is plastically deformed, under hydrostatic pressure, while the fragments are formed in the neighboring elastic zone. $^{20}$

- Stresses. The elastic field is created by a point force at the corner of the mother particle and stresses are distributed in the elastic region. ${ }^{21,22}$ The equation that links the distance from the corner, $r$, and the size of a fragment, $x$, is defined as

$$
x=\frac{3 \mu}{E_{\mathrm{col}}^{4 / 3} H_{\mathrm{v}}^{2 / 3}}\left(\frac{\Gamma}{K}\right) r^{4}
$$

where $E_{\mathrm{col}}$ is the collision energy [J], $\Gamma$ is the fracture resistance $\left[\mathrm{J} / \mathrm{m}^{2}\right], K$ is an efficiency parameter $[-]$, and $H_{\mathrm{v}}$ is the Vicker's hardness $[\mathrm{Pa}]$.

- Rittinger's law. The newly generated surface is proportional to the strain energy in the fragments. ${ }^{23,24}$ If Rittinger's law applies, the size of the fragment, $x$, can be related to the strain energy as ${ }^{25}$

$$
w x K=2 \Gamma
$$

where $w$ is the strain energy per unit volume $\left[\mathrm{J} / \mathrm{m}^{3}\right]$.
- Energy of collision. The collision energy depends on the mother particle mass, i.e., $\rho_{\mathrm{c}} k_{\mathrm{v}} \eta^{3}$, with $\eta$ being its characteristic dimension, and on the velocity of the colliding stirrer, $u_{\mathrm{col}}$ :

$$
E_{\mathrm{col}}(\eta)=\rho_{\mathrm{c}} k_{\mathrm{v}} \eta^{3} \frac{u_{\mathrm{col}}^{2}}{2}
$$

Typically, the collision velocity is the product of an efficiency, $\eta_{\mathrm{w}}$ and of the velocity of the tip of the stirrer, $u_{\text {tip }}$, which is calculated from operating variables as

$$
u_{\text {col }}=\eta_{\mathrm{w}} u_{\text {tip }}=\pi \eta_{\mathrm{w}}\left(\frac{\epsilon V_{\mathrm{sol}}}{P_{\mathrm{o}} d_{\mathrm{i}}^{2}}\right)^{1 / 3}
$$

where $\epsilon$ is the specific power input $[\mathrm{W} / \mathrm{kg}], V_{\text {sol }}$ is the volume of the solution $\left[\mathrm{m}^{3}\right], P_{0}$ is the power number of the stirrer [-], and $d_{\mathrm{i}}$ is the diameter of the impeller [m].

The hydrodynamics of the system has a strong influence on the value of the collision velocity. In this study, we assume that the efficiency $\left(\eta_{\mathrm{w}}\right)$ summarizes all the effect of fluid dynamics, and we take its value as reported by Mersmann. ${ }^{1}$ Other methods to calculate the value of the impact velocity have been proposed and their adoption would not modify the analysis that follows. ${ }^{26,27}$

In the following, a summary of the theory is presented, where the size of the mother particle impacting the stirrer is denoted as $\eta$ (a constant), and the size of the attrition fragments as $x$ (a distributed quantity).

The smallest fragment is formed in the elastic region closest to the corner of the crystal. Its expression results from the minimum surface that can be created according to Rittinger's law, and it purely depends on mechanical properties of the material: ${ }^{22}$

$$
x_{\min }=\frac{32}{3} \frac{\mu}{H_{\mathrm{v}}^{2}}\left(\frac{\Gamma}{K}\right)
$$

where $\mu$ is the shear modulus $\left[\mathrm{J} / \mathrm{m}^{3}\right]$.

By considering an energy balance between the collision energy and the energy to create a new surface (Rittinger's law), one can calculate the maximum distance from the corner at which a fragment can form, thus the maximum size of the fragment: ${ }^{1,22}$

$$
x_{\max }(\eta)=\frac{1}{2}\left(\frac{H_{\mathrm{v}}^{2 / 3} K}{\mu \Gamma}\right)^{1 / 3} E_{\text {col }}^{4 / 9}(\eta)
$$

This is dependent on the physical properties of the material, on the operating conditions, and on the size of the mother particle, through $E_{\text {col }}$ (see eq 22).

Given the geometry of the problem, by applying a material balance between the volume of fragments produced and the volume of the crystal in the elastic region, the following relation can be derived ${ }^{1}$

$$
k_{\mathrm{v}} N_{\mathrm{F}}(\eta) q_{0}(x, \eta)=\frac{\pi E_{\mathrm{col}}(\eta) H_{\mathrm{v}}^{1 / 2}}{9 \mu^{3 / 4}}\left(\frac{K}{\Gamma}\right)^{3 / 4} x^{-3.25}
$$

where $q_{0}(x, \eta)$ is the distribution of fragments, and $N_{\mathrm{F}}(\eta)$ the number of attrition fragments. The distribution of fragments is obtained from the normalization of eq 26 in the interval $\left[x_{\min }\right.$, $\left.x_{\max }\right]$ : 


$$
q_{0}(x, \eta)=\frac{2.25 x^{-3.25}}{x_{\min }^{-2.25}-x_{\max }^{-2.25}(\eta)} \quad x_{\min } \leq x \leq x_{\max }(\eta)
$$

By substituting the expression of $q_{0}(x, \eta)$ (eq 27) in eq 26, the total number of attrition fragments can be calculated as

$$
N_{\mathrm{F}}(\eta)=\frac{\pi}{21} \frac{H_{\mathrm{v}}^{1 / 2}}{k_{\mathrm{v}} \mu^{3 / 4}}\left(\frac{K}{\Gamma}\right)^{3 / 4}\left(x_{\min }^{-2.25}-x_{\max }^{-2.25}(\eta)\right) E_{\mathrm{col}}(\eta)
$$

and the total volume of attrition fragments

$$
V_{\mathrm{F}}(\eta)=N_{\mathrm{F}}(\eta) \int_{0}^{\eta} k_{\mathrm{v}} x^{3} q_{0}(x, \eta) \mathrm{d} x
$$

which is the third moment of the normalized attrition fragments distribution, eq 27, multiplied by the number of attrition fragments produced, eq 28 , and the volume shape factor, $k_{\mathrm{v}}$.

According to this model, the size of the mother particle, $\eta$, cannot be smaller than the value corresponding to a volume of fragments equal to 0 . By setting eq 29 to 0 , the minimum size of the mother particle can be calculated by solving $x_{\min }=x_{\max }\left(\eta_{\min }\right)$ for $\eta_{\min }$ and thus obtain

$$
\eta_{\min }=\left(\frac{64}{3}\right)^{3 / 4} \frac{\mu \Gamma}{H_{\mathrm{v}}^{5 / 3} \mathrm{~K}}\left(\frac{2}{\rho_{\mathrm{c}} k_{\mathrm{v}} u_{\mathrm{col}}^{2}}\right)^{3}
$$

Breakage Model - Secondary Nuclei As Breakage Fragments. The birth and death terms of eqs 1 and 5 result from the formation of fragments due to breakage. In this work, we assume that the only cause for breakage is the collision of crystals with the stirrer; thus, neither crystal-crystal nor crystal-wall collisions are explicitly considered.

In the following, we discuss the terms of eqs 5 , i.e., the breakage rate, and the daughter distribution, and we conclude with the constraints that the model of the attrition process has to fulfill.

The model presented here is the implementation of the theory of Gahn and Mersmann ${ }^{20}$ for a population balance equation coupled with the fragmentation mechanism, where the model of Iggland and Mazzotti ${ }^{18}$ is used.

Breakage Rate. The expression of the breakage frequency for a stirred tank reactor, according to Szilágyi and Lakatos, ${ }^{28}$ is

$$
b(x)=k_{\mathrm{b}} \frac{\rho_{\mathrm{i}} l_{\mathrm{i}}^{2} h_{\mathrm{i}} N_{\mathrm{i}}^{3} d_{\mathrm{i}}^{5}}{768 \pi} \zeta(x)
$$

where the subscript "i" refers to properties of the impeller, $\rho$ is its density, $l$ its width, $h$ its height, $d$ its diameter, and $N$ its rotation speed. The breakage rate constant $k_{\mathrm{b}}$ changes according to the type of breakage; the value for attrition from Szilgyi and Nagy ${ }^{29}$ is used here.

The factor $\zeta(x)$ takes into account that smaller particles break less easily than bigger ones. For this reason, the following functional dependence on the size of the particle has been suggested: ${ }^{30}$

$$
\zeta(x)=x\left(1+\exp \left(-\frac{x-k_{\mathrm{b}, 2}}{x_{\mathrm{ref}, 1}}\right)\right)^{-1}
$$

The parameter $k_{\mathrm{b}, 2}$ determines a range of sizes $x$, below which breakage no longer occurs. Typical values of $k_{\mathrm{b}, 2}$ and $x_{\mathrm{ref}, 1}$ used here are reported in Table 1.
Table 1. Operating Conditions for the Determination of the Breakage Rate, in Equations 31 and $32^{a}$

\begin{tabular}{cl} 
parameter & \multicolumn{1}{c}{ value } \\
$k_{\mathrm{b}}$ & $200 \mathrm{~s}^{2} / \mathrm{kg} / \mathrm{m}^{6}$ \\
$\rho_{\mathrm{i}}$ & $7850 \mathrm{~kg} / \mathrm{m}^{3}$ \\
$l_{\mathrm{i}}$ & $0.02 \mathrm{~m}$ \\
$h_{\mathrm{i}}$ & $0.002 \mathrm{~m}$ \\
$d_{\mathrm{i}}$ & $0.1 \mathrm{~m}$ \\
$N_{\mathrm{i}}$ & $10 \mathrm{rps}$ \\
$x_{\mathrm{ref}, 1}$ & $1 \times 10^{-6} \mathrm{~m}$ \\
$k_{b, 2}$ & $\eta_{\min }$ \\
$P_{0}$ & 0.3 \\
$V_{\text {sol }}$ & $5 \times 10^{-4} \mathrm{~m}^{3}$ \\
$\eta_{w}$ & 0.8 \\
$\epsilon$ & $5 \mathrm{~W} / \mathrm{kg}$
\end{tabular}

${ }^{a}$ The value of $k_{b, 2}$ is taken as the minimum size of the mother particle that can undergo attrition, $\eta_{\min }$ (see the Modeling Secondary Nucleation by Attrition section for clarifications).

Daughter Distribution. The daughter distribution in a breakage process specifies the number of fragments generated in the breakage event and their distribution in size. In this work, the daughter distribution is modeled as the sum of an attrition and a fragmentation contribution for reasons of (i) physical correctness, (ii) mathematical consistency, and (iii) numerical feasibility. A mother particle of size $\eta$ breaks according to the attrition daughter distribution, $g_{a}$, which produces the number and the volume of attrition fragments according to Mersmann's theory (see above). The expression of $g_{a}$ is the product of the number of attrition fragments (eq 28) and the normalized fragments distribution (eq 27):

$$
g_{\mathrm{a}}(x, \eta)=N_{\mathrm{F}}(\eta) q_{0}(x, \eta)
$$

Thus, the physical mechanism leads to the formation of $N_{\mathrm{F}}$ fragments, given by eq 33 , and a chipped particle of volume equal to that of the mother crystal minus $V_{\mathrm{F}}$ (eq 29).

A possible description of such a situation would entail writing the overall daughter distribution as the sum of $g_{\mathrm{a}}(x, \eta)$ (eq 33) and a Dirac delta generalized function centered in $\left(k_{\mathrm{v}} \eta^{3}-V_{\mathrm{F}}(\eta)\right)^{1 / 3}$. Though mathematically consistent, such approach would lead to big numerical difficulties requiring numerical approximations of the Dirac delta term.

Instead, we have decided to describe the chipped mother particle using the following two-particle U-shaped continuous distribution:

$$
g_{\mathrm{f}}(x, z)=3\left(2 k_{\mathrm{f}}+1\right) x^{2}\left(\frac{2}{z^{3}}\right)^{2 k_{\mathrm{f}}+1}\left(x^{3}-\frac{z^{3}}{2}\right)^{2 k_{\mathrm{f}}}
$$

where $z$ is the size of the breaking particle (in our case the physical chipped off mother crystal, i.e., $\left.z=\left(k_{\mathrm{v}} \eta^{3}-V_{\mathrm{F}}(\eta)\right)^{1 / 3}\right)$, and the parameter $k_{\mathrm{f}}$ affects the shape of the U-shaped function. Large values of $k_{\mathrm{f}}$ lead to two fragments very different in size, while $k_{\mathrm{f}}=0$ results in a constant daughter distribution. ${ }^{18}$ In this study, $k_{\mathrm{f}}$ is always taken equal to 50 , which implies that the large and the small fragment have size essentially equal to that of the chipped off mother particle and to zero, respectively.

The complete breakage process is illustrated in Figure 4; its final mathematical formulation is

$$
g(x, \eta)=g_{\mathrm{a}}(x, \eta)+g_{\mathrm{f}}\left(x,\left(k_{\mathrm{v}} \eta^{3}-V_{\mathrm{F}}(\eta)\right)^{1 / 3}\right)
$$




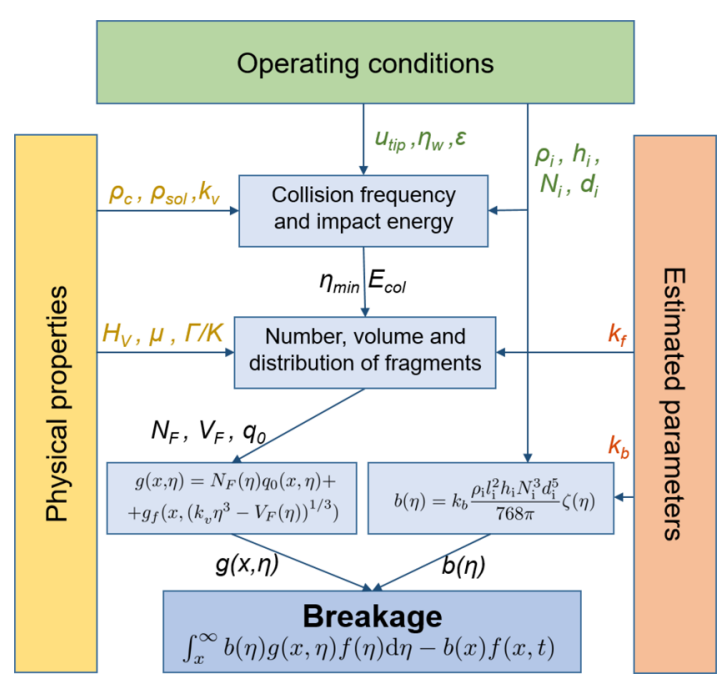

Figure 3. Mechanical properties of the crystals, physical properties of the system, operating conditions of the stirrer geometry and material, and estimated parameters playing a role in the definition of the attrition mechanism and their interplay.

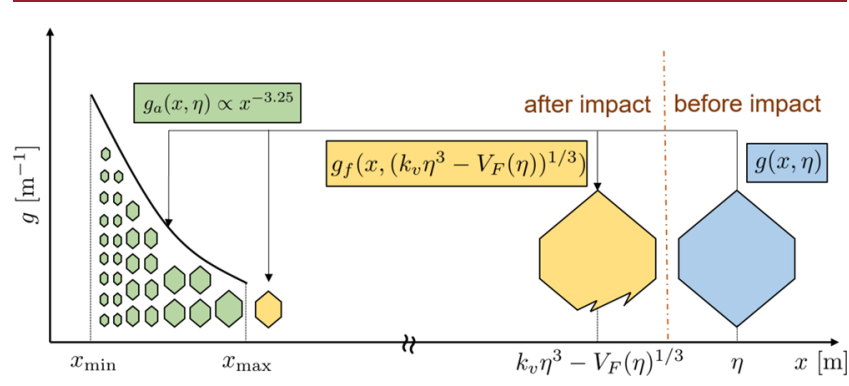

Figure 4. Scheme of the formation of daughter particles (green and yellow) and of the construction of the daughter distribution. $g_{\mathrm{a}}$ is the contribution of the attrition fragments, eq 33 in green, and $g_{\mathrm{f}}$ is the contribution of fragmentation, eq 34 in yellow.

It is worth noticing that usually the daughter distribution is defined independently of the operating conditions, which in turn play a role in determining the breakage rate constant. However, in this case, $g$ depends on the number and volume of fragments, as in Figure 3, so it cannot be determined without taking into account the operating conditions as well.

With reference to Figure 4, where a specific value of the critical crystal size $x_{c}$ is chosen for illustration purposes, it is worth noticing the following: fragments with $x<x_{\mathrm{c}}$ will tend to dissolve and disappear, whereas those with $x>x_{c}$ will tend to grow and survive.

Mass and Number Conservation. In a breakage process, the daughter distribution has to fulfill two physical constraints. The first is on the number of daughter particles formed at each breakage event, in our case:

$$
\int_{0}^{\eta}\left(g_{\mathrm{a}}(x, \eta)+g_{\mathrm{f}}\left(x,\left(k_{\mathrm{v}} \eta^{3}-V_{\mathrm{F}}(\eta)\right)^{1 / 3}\right) \mathrm{d} x=N_{\mathrm{F}}(\eta)+2\right.
$$

where every attrition event would result in the formation of $N_{F}(\eta)$ attrition fragments and every fragmentation event in the formation of two particles.

The second constraint is on the conservation of the total volume, i.e.:

$$
\int_{0}^{\eta} k_{\mathrm{v}} x^{3}\left(g_{\mathrm{a}}(x, \eta)+g_{\mathrm{f}}\left(x,\left(k_{\mathrm{v}} \eta^{3}-V_{\mathrm{F}}(\eta)\right)^{1 / 3}\right)\right) \mathrm{d} x=k_{\mathrm{v}} \eta^{3}
$$

where the volume of fragments formed by attrition, $V_{\mathrm{F}}(\eta)$, plus the volume of the two particles resulting from fragmentation has to be equal to the volume of the mother particle.

Nucleation Model - Secondary Nuclei As New Nuclei of Zero Size. With the nucleation model, we aim at embedding the Mersmann's model of secondary nucleation by attrition within the standard description of secondary nucleation as an event generating new nuclei of zero size, which appear in the PBE as a boundary condition at $x=0$ (eqs 3 and 6). This approach is simpler from a numerical point of view, but requires care because growth is size-independent (eq 14), and all new nuclei grow and contribute to the final PSD.

In order to do this, we focus on the physical meaning of the nucleation rate $J_{S}$ in eq 6 rather than on its standard functional dependence ( $r$ s of eq 6 ). $J_{S}$ gives the number of fragments of very small size generated via secondary nucleation (i.e., by attrition due to collision of mother crystals in the suspension with the stirrer according to Mersmann's model) per unit mass of solution and unit time. This is given by the product of (as illustrated schematically in Figure 5): (i) the number of mother

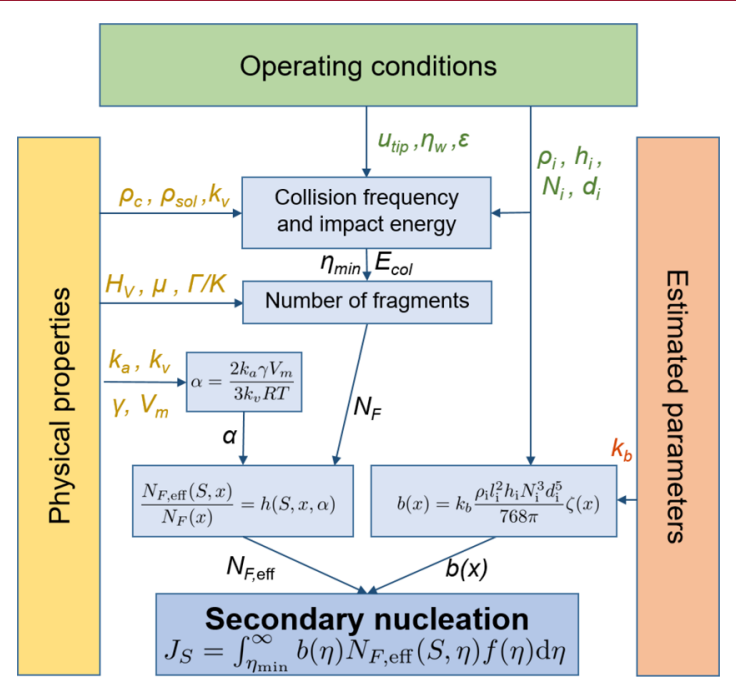

Figure 5. Physical properties, operating conditions, and estimated parameters playing a role in the definition of the secondary nucleation rate by attrition.

crystals in the suspension, which is given by all crystals in the suspension of size large enough to be able to undergo breakage by attrition, i.e., larger than $\eta_{\min }$ defined by eq 30 , and which is called $N_{\text {part; }}$

$$
N_{\text {part }}=V_{\text {sol }} \rho_{\text {sol }} \mu_{0}=V_{\text {sol }} \rho_{\text {sol }} \int_{\eta_{\text {min }}}^{\infty} f(x) \mathrm{d} x
$$

(ii) the frequency of the collisions, which is given by the sizedependent breakage rate constant of eq $31, b(x)$; (iii) the number of fragments that form in each collision and that survive because they are larger when they form than the critical size at the prevailing supersaturation conditions. It is clear that such definition leads to a secondary nucleation rate that depends on the crystals in suspension and their PSD, on the operating conditions in the crystallizer (energy input, impeller speed, etc.) 
and on the supersaturation, exactly as postulated by the empirical relationship given by the rhs of eq 6 .

The last term in the product above deserves two important remarks. First, only the $N_{F}$ attrition fragments given by the daughter distribution by attrition $g_{\mathrm{a}}(x, \eta)$ (eq 33) have to be considered; the mother crystal remains unchanged in this case because all fragments are assumed to have zero size. Second, with reference to Figure 4, only the attrition fragments larger than $x_{c}(S)$ (and smaller than $x_{\max }$ ) have to be included because they are the only ones that do not dissolve and finally survive. We call this number $N_{\mathrm{F}, \text { eff; }}$ it depends on the size of the mother crystal and on the supersaturation, and can be calculated as

$$
\frac{N_{\mathrm{F}, \mathrm{eff}}(S, x)}{N_{\mathrm{F}}(x)}=\frac{x_{\mathrm{c}}^{-2.25}(S)-x_{\max }^{-2.25}(x)}{x_{\min }^{-2.25}-x_{\max }^{-2.25}(x)}
$$

where the critical size $x_{\mathrm{c}}$ is defined by eq 8 . The dependency of $N_{\mathrm{F}, \text { eff }}$ on $x$ and on $S$ is illustrated in Figure 6. It is obvious that for

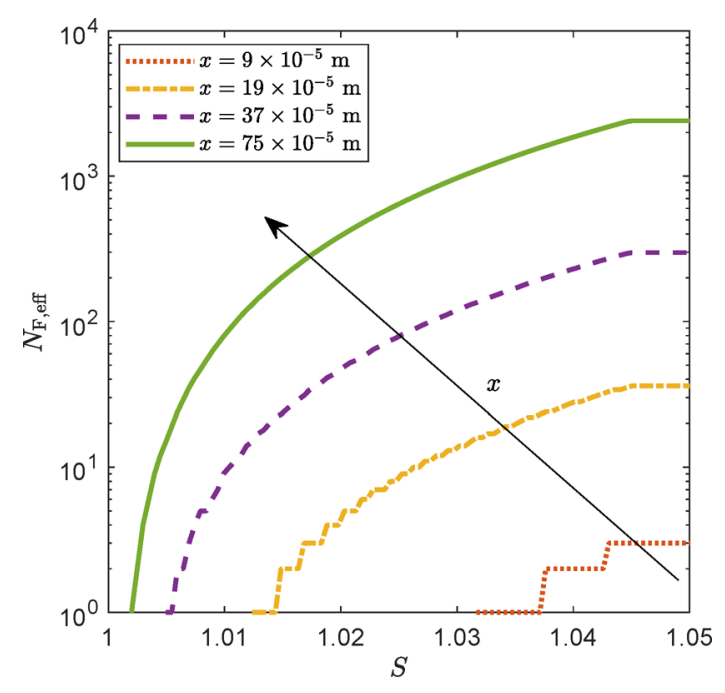

Figure 6. Dependencies of the effective number of fragments on supersaturation, $S$, and on the size of the mother particle, $x$. Given a mother particle size, e.g., $x=75 \times 10^{-5} \mathrm{~m}$ in green, moving toward high supersaturation leads to the formation of more fragments. For a fixed level of supersaturation, the smaller the mother particle, the smaller the number of fragments produced.

a given mother crystal the number of fragments increases with supersaturation (as normally accounted for also by the second term in the rhs of eq 6 and that at a given supersaturation smaller crystals yield less fragments by attrition).

According to the considerations above, the secondary nucleation rate is

$$
J_{S}(\theta, S)=\int_{\eta_{\min }}^{\infty} b(\theta, x) N_{\mathrm{F}, \text { eff }}(S, x) f(x) \mathrm{d} x
$$

\section{NUMERICAL METHODS}

Given the different phenomena that the two models are describing, two different numerical approaches are necessary. In particular, every phenomenon, i.e., growth and dissolution, attrition, and secondary nucleation, has been solved with a method that suits the physical nature of the phenomenon itself.

On the one hand, a finite volume method (FVM) has been implemented to solve growth and dissolution, ${ }^{31,32}$ since the method divides the size domains in cells, and then it calculates the fluxes from a cell to the adjacent ones. If the flux is toward the bigger sizes, it is interpreted as growth; otherwise, as dissolution. On the other hand, while solving breakage, the volume of particles has to be exactly conserved, and the number of particles formed has to be the one desired. The method of fixed pivot (FP) is able to fulfill these constraints. ${ }^{33}$ Finally, secondary nucleation can be described as a flux at the boundary; thus it can easily be integrated in a finite volume scheme.

In this section, the different numerical methods and their combination are described: first, the finite volume scheme is presented, then the fixed pivot, and finally the fractional-step method, i.e., a technique to combine the first two schemes. ${ }^{31}$

Finite Volume Method. Given a continuous function $n$ and a discrete size grid of size $M$, where the midcell points are $y_{i}$, with $i=1: M$, the value of the discrete function $N_{i}$ is calculated as

$$
N_{i}(t)=\frac{1}{y_{i+1 / 2}-y_{i-1 / 2}} \int_{y_{i-1 / 2}}^{y_{i+1 / 2}} n(\tilde{y}, t) \mathrm{d} \tilde{y}
$$

where the smaller the $\Delta y_{i}=y_{i+1 / 2}-y_{i-1 / 2}$, the smaller the error of interpreting the integral with its discretized form is, according to the central limit theory.

If we consider only the homogeneous problem, thus the left-handside of the population balance in eq 17, the FVM can be applied, as presented by Leveque $^{31}$ and Gunawan et al.: ${ }^{32}$

$$
\begin{aligned}
N_{i}^{m+1}= & N_{i}^{m}-\frac{\Delta \tau}{\Delta y}\left[\mathcal{F}\left((G N)_{i}^{m},(G N)_{i+1}^{m}\right)\right. \\
& \left.-\mathcal{F}\left((G N)_{i-1}^{m},(G N)_{i}^{m}\right)\right]
\end{aligned}
$$

where the part of the fluxes was resolved with a high-resolution method, e.g., Lax-Wendroff. ${ }^{31,32}$

Technical Details. In the following, technical details about the implementation of high-resolution methods, the boundary conditions and the Courant-Friedrichs-Lewy condition are presented.

- The solution of eq 42 could also be obtained by discretizing it with a finite difference method, but the flux limiter acts as an antidiffusive term, which sharpen the diffusive approximation. Flux-limiter methods can be linear, e.g., upwind, Lax-Wendroff, Beam-Wamring, Fromm, or high-resolution, e.g., minimod, superbee, MC, van Leer, etc. In this study, the method of van Leer has been implemented as presented by Leveque. ${ }^{31}$

- In order to facilitate the calculations at the boundaries, the domain has been extended to include two additional cells on either end, called ghost cells, whose values depend on the solution of the previous time step and on the boundary conditions. The values in the ghost cells are set at the beginning of each iteration step. By setting ghost cell values by extrapolation, the method becomes an upwind method at the boundaries.

- The Courant-Friedrichs-Lewy condition (CFL) is a necessary condition to ensure convergence while integrating the differential problem. It ensures that the time and size discretization are able to capture the change in population that happens at velocity $\max _{i}\left|G_{i, k}\right|$, in the case of size-dependent growth and dissolution. Such condition can be cast as

$$
\left(\max _{i}\left|G_{i, k}\right|\right) \Delta \tau \leq \Delta y
$$

The methods discussed so far have all been fully discrete methods, which means that the model is discretized in both space and time. Sometimes, it is useful to consider the discretization only in space, thus applying a semidiscrete scheme. We then integrate in time using any standard numerical method for systems of ordinary differential equations (ODEs).

Fixed Pivot. The part of eq 1 concerning breakage and death is solved using the method of fixed pivots. ${ }^{33}$ By applying this method, the zeroth and third moment of the particle distribution is conserved by construction. The discretized terms of birth and death due to the attrition process can be written as 


$$
B_{\mathrm{m}}-D_{\mathrm{m}}=K_{\mathrm{bg}}\left(\sum_{k=i}^{M} F_{\mathrm{m}, k} Q_{i, k} y_{k}^{\xi}-F_{\mathrm{m}, y_{i}^{\xi}}\right)
$$

where $K_{\mathrm{bg}}$ is the rescaling factor for the breakage term, $F_{m, k}$ is the discretized population at time $\tau_{m}$ and size $y_{k}$, and the quantity $Q_{i, k}$ defines the generic element of a matrix that, since it is timeindependent, can be calculated just once at the beginning of the simulation and used for all the following iterations:

$$
Q_{i, k}=\int_{y_{i-1}}^{y_{i}} \gamma_{i}^{(i-1)}(u) g\left(u, y_{k}\right) \mathrm{d} u+\int_{y_{i}}^{y_{i+1}} \gamma_{i}^{(i)}(u) g\left(u, y_{k}\right) \mathrm{d} u
$$

The expression of $\gamma_{i}$ is given in the work by Kumar and Ramkrishna, ${ }^{33}$ and $g$ is the daughter distribution.

Fractional-Step Method. In the presence of source terms, the problem is not homogeneous anymore, and other methods have to be implemented. In some cases, the source term can also be easily integrated; thus a finite volume scheme could be easily used. If the source term is more complicated, e.g., an integro-differential term as the one resulting from attrition, the literature ${ }^{31}$ suggests to use a fractionalstep method, in which the complete problem

$$
\frac{\partial f(x, t)}{\partial t}+\frac{\partial(g(x) f(x, t))}{\partial x}=h(f, t)
$$

is solved in two steps, whose combination can approximate the solution of the complete equation:

$$
\begin{aligned}
& \frac{\partial f(x, t)}{\partial t}+\frac{\partial(g(x) f(x, t))}{\partial x}=0 \\
& \frac{\partial f(x, t)}{\partial t}=h(f, t)
\end{aligned}
$$

In eqs 46 and $47, g$ is a generic growth function and $h$ a generic source term.

In the full problem with growth and breakage, the convective term (growth) and the source term (breakage) are solved with different methods. The iterative method has been implemented as a hybrid.

At each time step, e.g., $t_{\mathrm{m}}$, the homogeneous problem (eq 48) is solved with a finite volume scheme, which gives as a result a partial solution of the problem at the following time step, i.e., $\tilde{N}_{m+1}$ :

$$
\tilde{N}_{m+1}-N_{m}-\int_{t_{m}}^{t_{m+1}} \frac{\partial\left(G^{*}(S, y) n(y, \tau)\right)}{\partial y} \mathrm{~d} \tau=0
$$

This partial solution is used as the initial condition for the following ordinary differential problem, which is solved with a fixed pivot scheme.

$$
N_{m+1}-N_{m}=\int_{t_{m}}^{t_{m+1}}\left(B^{*}(y, \tau)-D^{*}(y, \tau)\right) \mathrm{d} \tau
$$

The result of this second equation is the solution at the time step $t_{m+1}$ i.e., $N_{m+1}$. The same procedure is iterated for the following time steps, where $N_{m+1}$ is used as initial condition to solve the homogeneous problem (eq 48) at the time step $t_{m+2}$.

The accuracy of the fractional-step method can be demonstrated only for relatively easy problems, where the solution can be calculated also analytically. For the considered equations, no analytical solution is available, so the only way to prove that the method gives correct solutions is by performing convergence tests, i.e., by varying the step size in both coordinates.

Numerical Considerations. The two models are solved with different numerical methods; thus the computational time to perform the simulations is also different. The simulations of the breakage model have been solved by applying a fractional step method: for each time step, the part of growth is solved according to a finite volume method, while the part of breakage is discretized in space according to the fixedpivot method and then integrated in time by any standard ODE solver, e.g., Matlab-built-in "ode15s". The simulation of the nucleation model has been obtained by following a fully discrete finite volume method, and the secondary nucleation rate is added as a boundary condition at every time step.

To solve a simulation similar to those presented below, the necessary computational time for the breakage model is longer than the one necessary to solve the same simulation with the nucleation model. The most expensive step is the calculation of the breakage matrix, which is essential for the implementation of the method of fixed pivot. An alternative could be the implementation of a finite volume scheme also for the breakage term, as reported by Kumar and Kumar, ${ }^{34}$ which might

\begin{tabular}{|c|c|c|}
\hline \multicolumn{2}{|l|}{ Breakage model } & Nucleation model \\
\hline $\begin{array}{l}\frac{\partial f}{\partial t}+\frac{\partial(G f)}{\partial x}=B-D \\
\frac{\mathrm{d} c}{\mathrm{~d} t}=-k_{v} \rho_{c} \frac{\mathrm{d} \mu_{3}}{\mathrm{~d} t}\end{array}$ & & $\begin{array}{l}\frac{\partial f}{\partial t}+G \frac{\partial f}{\partial x}=0 \\
\frac{\mathrm{d} c}{\mathrm{~d} t}=-k_{v} \rho_{c} \frac{\mathrm{d} \mu_{3}}{\mathrm{~d} t}\end{array}$ \\
\hline $\begin{array}{l}f(x, t=0)=f_{\text {init }}(x) \\
c(t=0)=c_{\text {init }} \\
f(x=0, t)=0 ;\end{array}$ & $\begin{array}{l}\text { Initial and } \\
\text { boundary } \\
\text { conditions } \\
\text { II }\end{array}$ & $\begin{array}{l}f(x, t=0)=f_{\text {init }}(x) \\
c(t=0)=c_{\text {init: }} \\
f(x=0, t)=J] G_{0}\end{array}$ \\
\hline$G=k_{g}\left(S-\exp \left(\frac{\alpha}{x}\right)\right.$ & $\begin{array}{l}\text { Constitutive } \\
\text { equations }\end{array}$ & $G=k_{g}(S-1)$ \\
\hline $\begin{aligned} B & =\int_{x}^{\infty} b(\eta) f(\eta, t) g(x \\
D & =b(x) f(x, t)\end{aligned}$ & 7) $\mathrm{d} \eta$ & $J_{S}=E_{t}(\theta) F_{1}(S) F_{2}(f)$ \\
\hline
\end{tabular}
be faster, but it would not ensure the conservation of both the exact number of particles and the total volume during the breakage process.

\section{RESULTS AND DISCUSSION}

In this section we analyze the advantages and disadvantages of the nucleation model and of the breakage model (see the summary of the corresponding equations in Figure 7). To this

Figure 7. Comparison between the two PBE models, with initial, boundary conditions, and constitutive equations. Both models describe secondary nucleation by attrition with two different approaches and by using different mathematical formulations.

aim, we have simulated a seeded growth crystallization process in an isothermal batch crystallizer in the presence of secondary nucleation using the two models, with model parameters and initial conditions that correspond exactly to the same physical situation. We have carried out also another simulation, using a third model, consisting of the breakage model during an initial phase, and then of the breakage model without secondary nucleation (see below for details, where it will be clarified that this corresponds to a physically realistic scenario).

In particular, the rescaling and growth parameters are always the same, namely, $x_{0}, k_{g, 1}, E_{g}, t_{0}$ calculated as in the Rescaled Model section and presented in Table 2 . The initial parameters of the simulations are the mean size of the particle size distribution, $\bar{x}_{\text {init }}=200 \mu \mathrm{m}$, its standard deviation, $\sigma_{\text {init }}=0.05 \bar{x}_{\text {init }}$ and its third moment, $\mu_{3, \text { init }}$ which has been estimated from a suspension density, $\rho_{\mathrm{D}}$, of $1 \%$ through eq 50 and then rescaled, according to eq 18 .

$$
\mu_{3, \text { init }}=\frac{\rho_{\mathrm{D}}}{k_{\mathrm{v}} \rho_{\mathrm{c}}}
$$

The simulations are all started from an initial supersaturation of $S_{\text {init }}=1.1$.

The simulation results are illustrated in Figures 8, 9, 10, and 11. In Figure 8 the temporal evolution of the zeroth moment of the PSDs calculated using the nucleation model (red solid line), the breakage model (blue solid line), and the third model (yellow solid line) is shown, together with the temporal 
Table 2. Physical Properties of Potassium Alum ${ }^{a}$

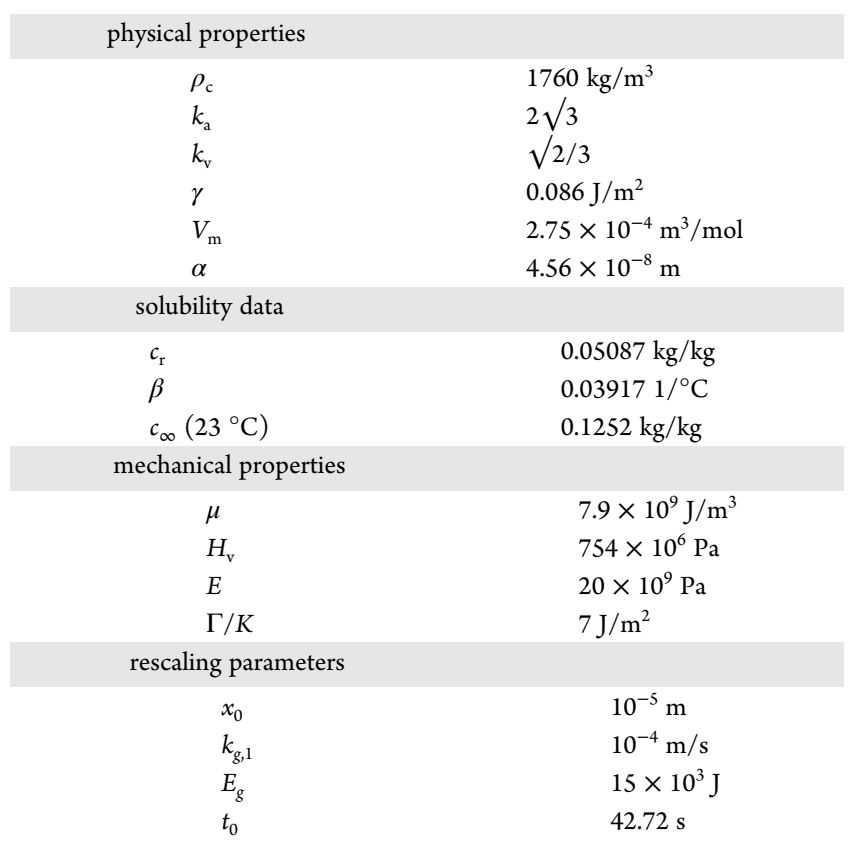

${ }^{a}$ Data for the determination of the capillary length, $\alpha$, at $23{ }^{\circ} \mathrm{C}$. The shape of potassium alum crystals is assumed to be octahedron. ${ }^{35}$ Solubility data of potassium alum in water. The parameters are fitted from gravimetric experiments on eq 10. Mechanical properties of potassium alum for the implementation of the attrition model. $\mu$ is the shear modulus, $H_{\mathrm{v}}$ the Vicker's hardness, $E$ the Young modulus, and $\Gamma / K$ the resistance to fracture. All the values are taken from Gahn and Mersmann. ${ }^{11}$ Rescaling and growth parameters used throughout the simulations. Their derivation and meaning can be found in the Rescaled Model section.

evolution of the supersaturation (green solid line, which is the same for all three PBE models). Note that the zeroth moment of the PSD is the number of particles and that we use a logarithmic time scale for the sake of clarity. Figures 9-11 show the PSDs obtained with the different models at different meaningful time instants during the process. Since, due to secondary nucleation, the PSD is in many cases bimodal, i.e., it consists of the initial

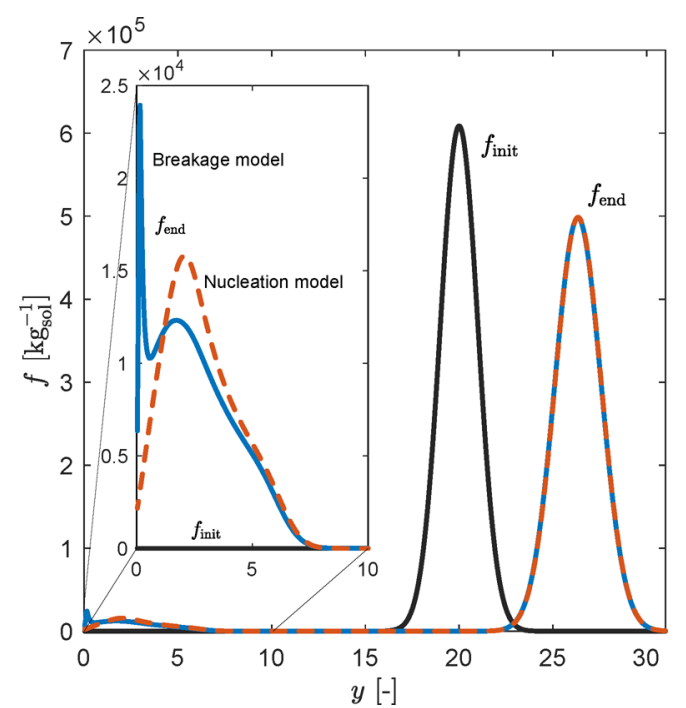

Figure 9. PSD at the beginning and at the end of the seeded growth stage. The big particles of $f_{\text {init }}$ grow in the same way for both models. The population of fines (zoom on the lhs) is slightly different, since the breakage model is constantly producing fragments at very small sizes, but their mean size is almost the same.

seeds (that have grown to larger sizes) and of the secondary nuclei (fines), in all figures the main plot shows the grown seeds, whereas the inset is a zoom-in of the fines.

Let us consider Figure 8 first. It can be readily observed that in terms of supersaturation evolution, the simulation can be subdivided in two phases, namely, the first during which supersaturation is consumed (due to crystal growth) and the second where the solution is essentially saturated ( $S$ indistinguishable from 1). In terms of the evolution of the number of crystals in the PSD though, the second phase above is in turn subdivided in three phases, that will be called (i) early Ostwald ripening, (ii) aging, and (iii) late Ostwald ripening.

First Phase: Seeded Growth. Let us consider the part of the simulation from the beginning to when the supersaturation reaches a lower threshold, where crystal growth can be considered to be completed (without loss of generality we

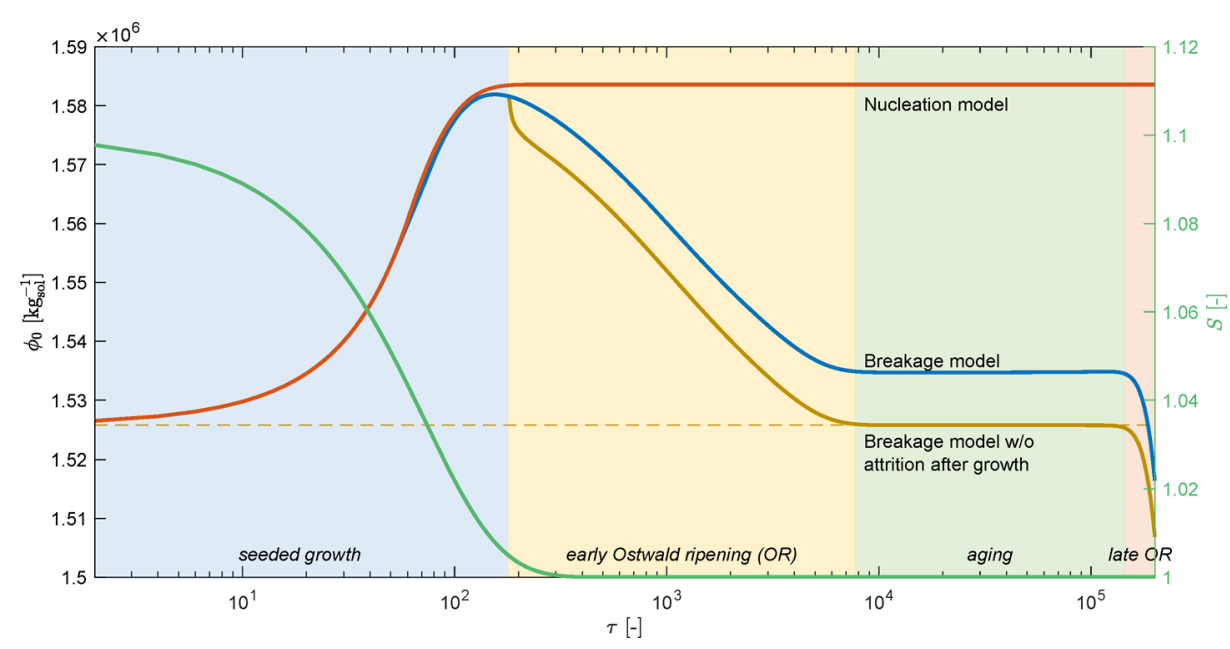

Figure 8. Evolution of the supersaturation (green, axis on the rhs) and of the zeroth moment, $\phi_{0}$ (axis on the lhs). The nucleation model (red) is producing particles, and its initial population is growing in the first phase. At low values of $S$, the PSD remains unchanged. The model of breakage (blue) and a variation on that, which should mimic the practical implementation of an industrial process (yellow), present a more complicated behavior, since at low $S$ size-dependent growth modifies the PSD, and it induces different stages of ripening. 


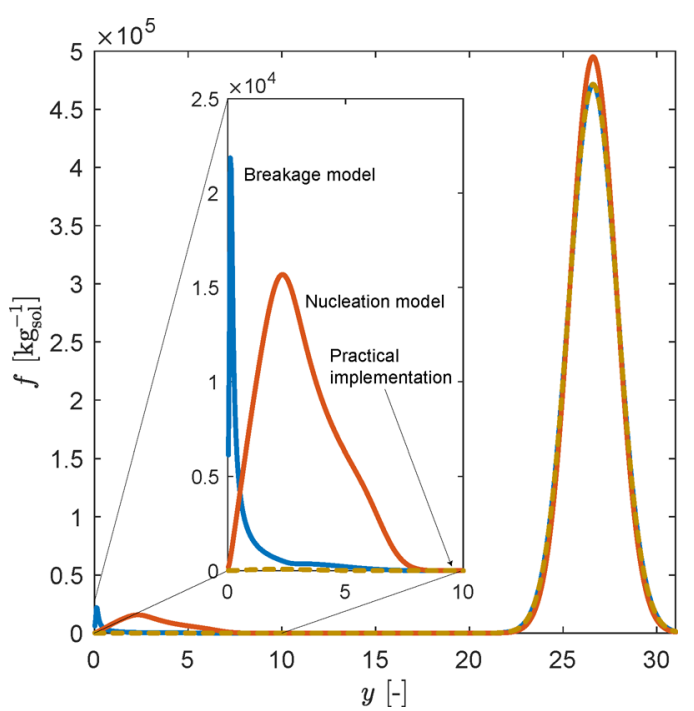

Figure 10. PSD at the end of the early Ostwald ripening stage. The PSD resulting from the nucleation model (red) does not change because of the small values of $S$. The breakage model (blue) has a smaller number of fines, but the peak at very small sizes is still present. The example of the practical implementation (yellow) shows that all the fines are dissolved in this phase; thus early ripening is completed, and the number of particles is back to the same value as the initial one.

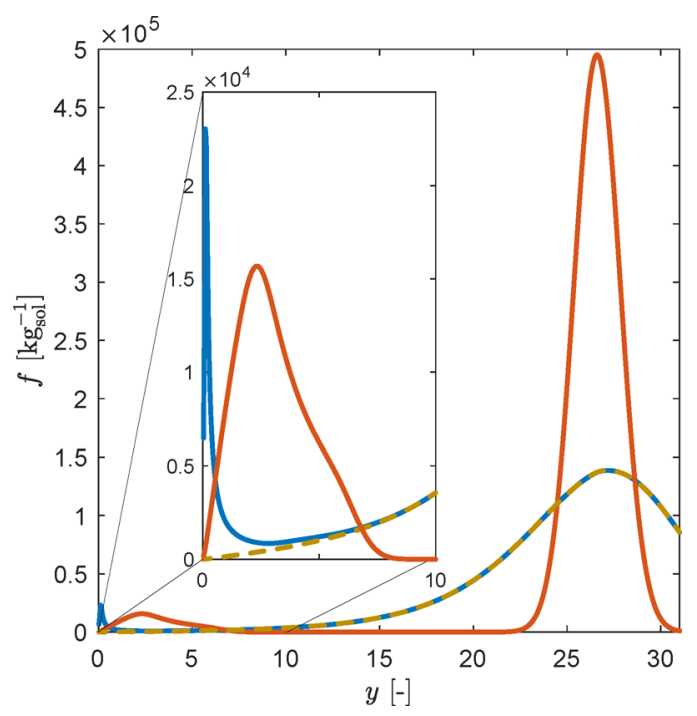

Figure 11. PSD during late Ostwald ripening. The main peaks of the populations (blue and yellow) have spread across the entire space domain, because of size-dependent solubility: bigger particles are growing at the expense of small particles that are dissolving.

have arbitrarily taken a value of 1.005). Figure 8 shows that during the growth phase secondary nucleation is active for all models, and that the zeroth moment of the PSD increases to its maximum value when the supersaturation attains the threshold value. The breakage model and the third model yield the same results by definition; the nucleation model yields very similar results. When considering also Figure 9, where the PSDs at the end of the growth phase are shown, we can notice that the initial seeds have grown in the same way for both models, with the mode of the PSD after growth, which is about 30\% larger than that of the initial seeds. On the other hand, the fines exhibit slightly different PSDs, which reflect the different implementations of secondary nucleation in the two models; interestingly, the integral of the PSD of the fines is very similar in the two cases.

Thus concluding, it is apparent that the nucleation model and the breakage model, which implement the same attrition model as proposed in this work, yield the same results during the seeded growth phase, or in other words, they deliver results that exhibit a difference that is smaller than the uncertainty in any experimental measurements. Therefore, the two models can be used indifferently as far as results are concerned, whereas they exhibit major numerical differences, the nucleation model being much less computationally intensive than the breakage model (see above for an in-depth discussion of numerical aspects).

Second Phase: Ripening and Aging of Crystals. Let us consider the second phase of the simulation, i.e., from when the supersaturation reaches its threshold level to the end. The third model differs from the breakage model, insofar as the secondary nucleation mechanism is switched off when $S$ drops below the threshold value of 1.005. The interest in this situation and the justification for this third simulation are as follows: there are indeed crystal formation processes, e.g., in the pigment industry, where, after growth under stirring, crystals are left ripen under different conditions, i.e., suspended in a saturated solution where attrition and secondary nucleation by attrition are not active because of lack of intense stirring. ${ }^{36}$

During the second phase of the simulation, the supersaturation approaches quickly the value of one, which has the consequence that the critical crystal size given by eq 8 becomes larger and larger. First, it becomes larger than the size of the fines, and then it becomes larger than the smaller crystals in the distribution of crystals stemming from the original seeds. Such a feature is irrelevant in the framework of the nucleation model, where solubility is size-independent. However, it is of crucial importance in the framework of the breakage model, where solubility is size-dependent, and crystals below the critical size dissolve.

As a consequence, with reference again to Figure 8, when the solution approaches saturation, the nucleation model does not evolve anymore; the PSD shown in Figure 9 (red solid line) is frozen and represents the final outcome of the process.

On the contrary, according to the breakage model and to the third model, the PSD keeps evolving under the effect of the driving force consisting of the difference between the solution concentration and the solubility of each crystal, which is determined by its specific size. Thus, during early Ostwald ripening fines dissolve, while the original seeds grow at a negligible rate, being the nominal supersaturation essentially one. This effect yields on the one hand the decrease of the zeroth moment of the distributions observed in Figure 8 and the disappearance of the fines in the case of the PSD calculated with the third model (see inset in Figure 10, yellow dashed line).

The difference in the case of the breakage model, where secondary nucleation is still active during ripening and aging, is that new fines keep forming, while those formed earlier are dissolving. This difference explains why the two profiles in Figure 8 differ during early Ostwald ripening, and why the zero-th moment calculated with the third model, where all the fines formed through secondary nucleation during seeded growth dissolve, attains at the beginning of the aging phase the same value as at the beginning of the process. It is worth noting that the zeroth moment calculated with the breakage model attains a constant value (at the beginning of the aging phase) when the loss of particles through dissolution and the formation of new secondary nuclei balance each other (this is also the reason of 
the different distribution of fines in the inset of Figure 10, blue solid line). Moreover, at the end of the early Ostwald ripening phase the PSD calculated by the third model, i.e., the breakage model without secondary nucleation, is again unimodal, with the seeds grown at their maximum size possible and their PSD as narrow as that of the seeds. A similar result has been described earlier for a model without attrition (see Figure $6 \mathrm{~d}$ in Iggland and Mazzotti ${ }^{18}$ ).

Thus concluding, the breakage model and the third model, thanks to size-dependent solubility, are able to describe ripening, which is important in some cases and cannot be described through the simpler nucleation model. The additional computational burden incurred by the breakage model (and by the third model), which is due to the presence of the integral breakage terms and to the fact that the growth factor in the PBE can be either positive (growth) or negative (dissolution), is the price that we have to pay to be able to model Ostwald ripening.

During aging, i.e., the second time period during the second phase of the process, the zeroth moment in Figure 8 remains constant. There is no net loss of crystals, because in the case of the third model, all fines have been dissolved and initial seeds are too large to dissolve completely, and in the case of the breakage model secondary nucleation and dissolution balance each other. The net effect of the aging phase is that the PSD of the large crystals stemming from the initial seeds becomes broader and broader because of ripening under conditions where the critical nucleus size is within the range of the distribution of the large crystals. Therefore, the lhs tail of the distribution is dissolving (partially), while the rhs tail is growing. This phenomenon ages the crystal population, in the sense that it leads to a powder with less attractive properties for applications than at the end of early Ostwald ripening (see Figure 11).

The onset of the late Ostwlad ripening phase occurs when the lhs tail of the distribution of large crystals reaches size zero, and crystals that were part of the initial seed population start to dissolve completely and to disappear. This leads to the rather fast drop of the zeroth order moment calculated by the breakage model and by the third model as observed in Figure 8. During late Ostwald ripening, the population of crystals should in principle evolve to the disappearance of all crystals but one, i.e., a state that cannot be described by a PBE model.

\section{CONCLUSIONS}

The description of secondary nucleation by attrition is of particular interest, since it is the most common source of fragments when operating a stirred tank reactor, ${ }^{3}$ in a batch or a continuous set up. In fact, the stirrer, by hitting the crystals, produces a large number of attrition fragments that then will grow or dissolve, according to the level of supersaturation in the system. In our case and in agreement with the mechanistic theory of Gahn and Mersmann, ${ }^{20}$ we consider these fragments as secondary nuclei. Two PBE models to describe secondary nucleation by attrition have been derived. The models are valid under certain assumptions, and they describe the attrition process, by using physical and mechanical properties of the crystals and a very limited number of estimated parameters.

The novelty of this contribution is how the attrition model is implemented, i.e., as a breakage term in the population balance equation and as a boundary condition, and the comparison between the two approaches. In the breakage model, attrition fragments are distributed in size, and their growth or dissolution is determined by size-dependent solubility. In the nucleation model, attrition fragments are produced as a boundary condition at size 0 according to an expression that is already taking into account the effect of supersaturation; thus, all the fragments produced will grow to full size crystals, and their growth rate is size-independent.

The numerically efficient solution of the population balance equations exploits the combination of different numerical methods, i.e., fixed pivot and finite volume methods.

Starting from the same conditions, both models give very similar results in the regime of high supersaturation, i.e., during the growth of seeds, even though the mathematical approach, and the numerical solution is different. At low values of $S$, sizedependent solubility is the only mechanism still active, which produces a series of effects, e.g., Ostwald ripening, visible only in the breakage model, which has size-dependent growth. The two models are also compared with a two-step version of the breakage model, which describes what might represent an example of practical implementation of a process involving attrition and ripening.

This paper presents three important novel elements. First, at a conceptual level, we have reconciled the fundamentally sound, physically based, and experimentally validated description of secondary nucleation by attrition proposed by Gahn and Mersmann with a mathematically correct population balance description of crystal growth in the presence of secondary nucleation. We have done this in two different ways, and we have discussed the similarities and differences of the two approaches, thus highlighting how these depend on the prevailing mechanism during crystallization (typically controlled by the level of supersaturation and stirring intensity).

Second, in the context of the breakage model where solubility is not assumed to be size-independent but it is properly considered to be size-dependent, we could bridge the gap between nucleation and ripening by being able to describe both with the same population balance model, which evolves in a seamless manner from a regime dominated by growth and secondary nucleation to one where the crystal population undergoes ripening and aging.

Finally, from an application perspective, we have developed efficient numerical algorithms for both models and highlighted numerical challenges, particularly in the case of the breakage model. Our analysis enables the researcher interested in modeling, for instance, continuous crystallization processes to choose the model which attains the best trade-off between accuracy in the physical description of the phenomena of interest and efficiency of the numerical algorithm.

\section{ASSOCIATED CONTENT}

\section{S Supporting Information}

The Supporting Information is available free of charge at https://pubs.acs.org/doi/10.1021/acs.cgd.9b01240.

Details about the implementation of breakage and secondary nucleation rate in the rescaled model; an additional secondary nucleation rate expression (PDF)

\section{AUTHOR INFORMATION}

\section{Corresponding Author}

*E-mail: marco.mazzotti@ipe.mavt.ethz.ch. Phone: +41 44632

24 56. Fax: +41446321141.

ORCID

Luca Bosetti: 0000-0001-6657-6510

Marco Mazzotti: 0000-0002-4948-6705 


\section{Notes}

The authors declare no competing financial interest.

\section{ACKNOWLEDGMENTS}

The authors thank Brigitta Bodák, ETH Zurich, and Giovanni Maria Maggioni, Georgia Tech, for the fruitful discussions. This project has received funding from the European Research Council (ERC) under the European Unions Horizon 2020 Research and Innovation Programme under Grant Agreement No. 2-73959-18.

\section{- LIST OF SYMBOLS FOR GROWTH AND SECONDARY NUCLEATION}

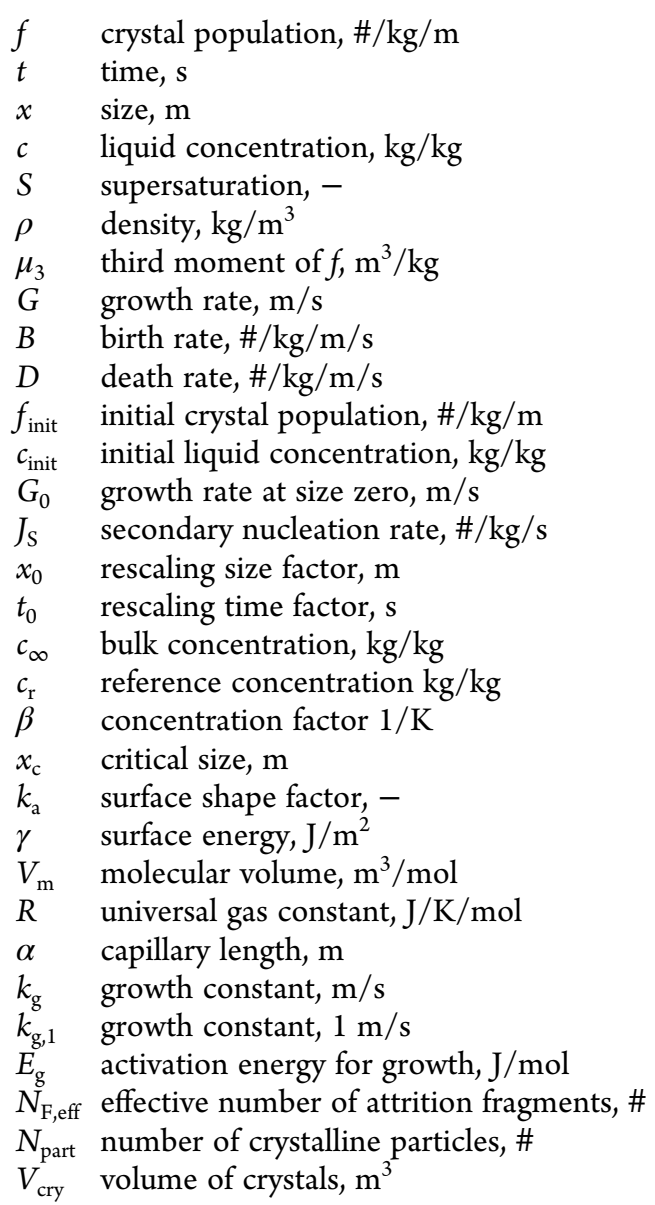

\section{LIST OF SYMBOLS FOR BREAKAGE}

$b$ breakage rate, \#/s

$k_{\mathrm{b}} \quad$ breakage rate constant, $\mathrm{s}^{2} / \mathrm{kg} / \mathrm{m}^{6}$

$\rho_{\mathrm{i}} \quad$ impeller density, $\mathrm{kg} / \mathrm{m}^{3}$

$l_{\mathrm{i}} \quad$ impeller length, $\mathrm{m}$

$h_{\mathrm{i}} \quad$ impeller height, $\mathrm{m}$

$d_{\mathrm{i}} \quad$ impeller diameter, $\mathrm{m}$

$N_{\mathrm{i}} \quad$ impeller revolution speed, $1 / \mathrm{s}$

$x_{\text {ref,1 }}$ reference size $1, \mathrm{~m}$

$k_{\mathrm{b}, 2} \quad$ size of minimum breakage, $\mathrm{m}$

$g$ daughter distribution, \#/m

$g_{a}$ attrition daughter distribution, \#/m

$g_{\mathrm{f}} \quad$ fragmentation daughter distribution, \#/m

$\eta \quad$ mother particle size, $\mathrm{m}$

$\mu$ shear modulus, $\mathrm{J} / \mathrm{m}^{3}$

$H_{v} \quad$ Vicker's hardness, $\mathrm{Pa}$

E Young modulus, $\mathrm{Pa}$
$\Gamma / K$ resistance to fracture, $\mathrm{J} / \mathrm{m}^{2}$

$E_{\text {col }}$ energy of collision, $\mathrm{J}$

$u_{\text {tip }}$ tip stirrer velocity $\mathrm{m} / \mathrm{s}$

$\eta_{\mathrm{w}} \quad$ collision efficiency, -

$u_{\text {col }}$ collision velocity, $\mathrm{m} / \mathrm{s}$

$P_{\mathrm{o}} \quad$ reactor power number, -

$V_{\text {sol }}$ solution volume, $\mathrm{m}^{3}$

$\rho_{\text {sol }}$ solution density, $\mathrm{kg} / \mathrm{m}^{3}$

$\epsilon \quad$ specific power input, $\mathrm{W} / \mathrm{kg}$

$\rho_{\mathcal{c}}, \quad$ crystal density $\mathrm{g} / \mathrm{m}^{3}$

$k_{\mathrm{v}} \quad$ volumetric shape factor, -

$x_{\min }$ minumum attrition fragment size, $\mathrm{m}$

$x_{\max }$ maximum attrition fragment size, $\mathrm{m}$

$q_{0} \quad$ normalized attrition fragment distribution, \#/m

$N_{\mathrm{F}} \quad$ number of attrition fragments, \#

$V_{\mathrm{F}} \quad$ volume of attrition fragments, $\mathrm{m}^{3}$

$\eta_{\text {min }}$ minimum size of mother particle, $\mathrm{m}$

$k_{\mathrm{f}} \quad$ fragmentation constant, -

\section{REFERENCES}

(1) Mersmann, A. Crystallization Technology Handbook; CRC Press, 2001.

(2) Kashchiev, D. Nucleation: Basic Theory with Application; Butterworth-Heinemann, 2000.

(3) Agrawal, S. G.; Paterson, A. H. J. Secondary nucleation: mechanisms and models. Chem. Eng. Commun. 2015, 202, 698-706.

(4) Randolph, A. D.; Larson, M. A. Theory of Particulate Processes; Acad. Press: New York, 1988.

(5) Zhang, D.; Wang, X.; Ulrich, J.; Tang, W.; Xu, S.; Li, Z.; Rohani, S.; Gong, J. Control of crystal properties in a mixed-suspension mixedproduct removal crystallizer: general methods and the effects of secondary nucleation. Cryst. Growth Des. 2019, 19, 3070-3084.

(6) Hulburt, H. M.; Katz, S. Some problems in particle technology. Chem. Eng. Sci. 1964, 19, 555-574.

(7) Botsaris, G. D. Secondary nucleation-a review. In Industrial Crystallization; Springer: Boston, MA, 1976; pp 3-22.

(8) Schönert, K.; Steier, K. Die Grenze der Zerkleinerung bei kleinen Korngrössen. Chem. Ing. Tech. 1971, 43, 773-777.

(9) Chianese, A.; Di Berardino, F.; Jones, A. G. On the effect of secondary nucleation on the crystal size distribution from a seeded batch crystallizer. Chem. Eng. Sci. 1993, 48, 551-560.

(10) Chianese, A.; Sangl, R. G.; Mersmann, A. B. On the size distribution of fragments generated by crystal collisions. Chem. Eng. Commun. 1996, 146, 1-12.

(11) Gahn, C.; Mersmann, A. Brittle fracture in crystallization processes Part A. Attrition and abrasion of brittle solids. Chem. Eng. Sci. 1999, 54, 1273-1282.

(12) Gahn, C.; Mersmann, A. Brittle fracture in crystallization processes Part B. Growth of fragments and scale-up of suspension cyrstallizers. Chem. Eng. Sci. 1999, 54, 1283-1292.

(13) Bermingham, S. K. A design procedure and predictive models for solution crystallisation processes. Ph.D. thesis, T.U. Delft, 2003.

(14) Iggland, M.; Mazzotti, M. Population balance modeling with sizedependent solubility: Ostwald ripening. Cryst. Growth Des. 2012, 12, $1489-1500$

(15) Ståhl, M.; Åslund, B.; Rasmuson, Å. C. Aging of reactioncrystallized benzoic acid. Ind. Eng. Chem. Res. 2004, 43, 6694-6702.

(16) Lifshitz, I. M.; Slyozov, V. V. The kinetics of precipitation from supersaturated solid solutions. J. Phys. Chem. Solids 1961, 19, 35-50.

(17) Wagner, C. Theorie der Alterung von Niederschlägen durch Umlösen. Z. Elektrochem. 1961, 65, 581-591.

(18) Iggland, M.; Mazzotti, M. A population balance model for chiral resolution via Viedma ripening. Cryst. Growth Des. 2011, 11, 46114622.

(19) Bodák, B.; Maggioni, G. M.; Mazzotti, M. Population-based mathematical model of solid-state deracemization via temperature cycles. Cryst. Growth Des. 2018, 18, 7122-7131. 
(20) Gahn, C.; Mersmann, A. Theoretical prediction and experimental determination of attrition rates. Chem. Eng. Res. Des. 1997, 75, 125-131.

(21) Michell, J. H. On distributions of stress in three dimensions. Proceedings of the London Mathematical Society 1900, s1-32, 23-61.

(22) Gahn, C. Die Festigkeit von Kristallen und ihr Einfluss auf die Kinetik in Suspensionskristallisatoren. Ph.D. thesis, TU Muenchen, 1997.

(23) Rumpf, H. Physical aspects of comminution and new formulation of a law of comminution. Powder Technol. 1973, 7, 145-159.

(24) Yoffe, E. H. Elastic stress fields caused by indenting brittle materials. Philos. Mag. A 1982, 46, 617-628.

(25) von Rittinger, P. Lehrbuch der Aufbereitungskunde in Ihrer Neuesten Entwicklung Und Ausbildung Systematisch Dargestellt; Ernst \& Korn, 1867.

(26) Rielly, C. D.; Marquis, A. J. A particle's eye view of crystallizer fuid mechanics. Chem. Eng. Sci. 2001, 56, 2475-2493.

(27) Tyrrell, R.; De Souza, B.; Frawley, P. J. Particle breakage: limiting conditions for crystal-crystallizer collisions. Cryst. Growth Des. 2018, 18, $617-622$.

(28) Szilágyi, B.; Lakatos, B. G. Model-based analysis of stirred cooling crystallizer of high aspect ratio crystals with linear and nonlinear breakage. Comput. Chem. Eng. 2017, 98, 180-196.

(29) Szilágyi, B.; Nagy, Z. K. Population balance modeling and optimization of an integrated batch crystallizer-wet mill system for crystal size distribution control. Cryst. Growth Des. 2018, 18, 1415.

(30) Salvatori, F.; Mazzotti, M. Experimental characterization and mathematical modeling of breakage of needle-like crystals in a continuous rotor-stator wet mill. Cryst. Growth Des. 2018, 18, 59575972.

(31) LeVeque, R. J. Finite Volume Methods for Hyperbolic Problems; Cambridge University Press, 2002; Vol. 31.

(32) Gunawan, R.; Fusman, I.; Braatz, R. D. High resolution algorithms for multidimensional population balance equations. AIChE J. 2004, 50, 2738-2749.

(33) Kumar, S.; Ramkrishna, D. On the solution of population balance equations by discretization-I. A fixed pivot technique. Chem. Eng. Sci. 1996, 51, 1311-1332.

(34) Kumar, R.; Kumar, J. Numerical simulation and convergence analysis of a finite volume scheme for solving general breakage population balance equations. Appl. Math. Comput. 2013, 219, 51405151.

(35) Kovačević, T.; Schock, J.; Pfeiffer, F.; Briesen, H. Shape identification of primary particles in potash alum aggregates using three-dimensional tomography data. Cryst. Growth Des. 2016, 16, 2685-2699.

(36) Myerson, A. S.; Erdemir, D.; Lee, A. Y. Handbook of Industrial Crystallization; Cambridge University Press, 2019. 